\title{
Usability Studies of an Egocentric Vision-Based Robotic Wheelchair
}

\author{
MOHAMMED KUTBI, Saudi Electronic University, Saudi Arabia \\ XIAOXUE DU, Teachers College, Columbia University \\ YIZHE CHANG, California State Polytechnic University \\ BO SUN, Stevens Institute of Technology \\ NIKOLAOS AGADAKOS, University of Illinois at Chicago \\ HAOXIANG LI and GANG HUA, Wormpex AI Research \\ PHILIPPOS MORDOHAI, Stevens Institute of Technology
}

\begin{abstract}
Motivated by the need to improve the quality of life for the elderly and disabled individuals who rely on wheelchairs for mobility, and who may have limited or no hand functionality at all, we propose an egocentric computer vision based co-robot wheelchair to enhance their mobility without hand usage. The robot is built using a commercially available powered wheelchair modified to be controlled by head motion. Head motion is measured by tracking an egocentric camera mounted on the user's head and faces outward. Compared with previous approaches to hands-free mobility, our system provides a more natural human robot interface because it enables the user to control the speed and direction of motion in a continuous fashion, as opposed to providing a small number of discrete commands. This article presents three usability studies, which were conducted on 37 subjects. The first two usability studies focus on comparing the proposed control method with existing solutions while the third study was conducted to assess the effectiveness of training subjects to operate the wheelchair over several sessions. A limitation of our studies is that they have been conducted with healthy participants. Our findings, however, pave the way for further studies with subjects with disabilities.

CCS Concepts: • Social and professional topics $\rightarrow$ Assistive technologies; $\bullet$ Computing methodologies $\rightarrow$ Computer vision; Computer vision tasks; $\bullet$ Human-centered computing $\rightarrow$ Interactive systems and tools;
\end{abstract}

Additional Key Words and Phrases: Wheelchair, egocentric camera

This research was primarily funded by National Institute of Nursing Research of the National Institutes of Health under Award Number R01NR015371 and also partially supported by the National Science Foundation under Awards IIS-1527294 and IIS-1637761. The content is solely the responsibility of the authors and does not necessarily represent the official views of the National Institutes of Health or the National Science Foundation.

Authors' addresses: M. Kutbi, 2624 Rayhanat Al Jazirah, Al Faisaliyyah, Jeddah 23442, Saudi Arabia; email: m.kutbi@seu.edu.sa; X. Du, Teachers College, Columbia University, 525 W 120th St, New York, NY 10027, USA; email: xd2164@tc.columbia.edu; Y. Chang, Mech Eng Dept, Cal Poly Pomona, 3801 West Temple Avenue, Pomona, California 91768, USA; email: chang@cpp.edu; B. Sun and P. Mordohai, Stevens Institute of Technology, Castle Point on Hudson, Hoboken, NJ 07030, USA; emails: \{bsun7, Philippos.Mordohai\}@stevens.edu; N. Agadakos, 1200 W Harrison St, Chicago, IL 60607, USA; email: nagad2@uic.edu; H. Li and G. Hua, Wormpex AI Research, 500 108th AVE NE, Suite 1740, Bellevue, WA 98004, USA; emails: \{oulhxustcer, ganghua\}@gmail.com.

Permission to make digital or hard copies of all or part of this work for personal or classroom use is granted without fee provided that copies are not made or distributed for profit or commercial advantage and that copies bear this notice and the full citation on the first page. Copyrights for components of this work owned by others than the author(s) must be honored. Abstracting with credit is permitted. To copy otherwise, or republish, to post on servers or to redistribute to lists, requires prior specific permission and/or a fee. Request permissions from permissions@acm.org.

(C) 2020 Copyright held by the owner/author(s). Publication rights licensed to ACM.

2573-9522/2020/07-ART4 \$15.00

https://doi.org/10.1145/3399434

ACM Transactions on Human-Robot Interaction, Vol. 10, No. 1, Article 4. Publication date: July 2020. 


\section{ACM Reference format:}

Mohammed Kutbi, Xiaoxue Du, Yizhe Chang, Bo Sun, Nikolaos Agadakos, Haoxiang Li, Gang Hua, and Philippos Mordohai. 2020. Usability Studies of an Egocentric Vision-Based Robotic Wheelchair. ACM Trans. Hum.Robot Interact. 10, 1, Article 4 (July 2020), 23 pages.

https://doi.org/10.1145/3399434

\section{INTRODUCTION}

Powered wheelchairs are among the most commonly used assistive devices, especially for people with certain motor impairments. An estimated $1 \%$ of the world's population requires a wheelchair, regardless of whether they have access to one. According to the 2010 census, there are 3.6 million wheelchair users in the US, while approximately $49 \%$ of older adults in Canadian institutional settings use a wheelchair [44]. Wheelchair users in Europe are estimated to be in the 5 million range, with 2 million of these users suffering from reduced upper-limb motor control and having to control their wheelchairs via alternative interfaces [6]. Different studies have shown that $10 \%$ of wheelchair users require help while operating their manually-controlled wheelchairs, and around $40 \%$ of users had difficulties in steering and maneuvering tasks using a powered wheelchair [11].

Most powered wheelchairs on the market are designed to be controlled through a joystick. However, people who have limited or no upper limb mobility, for instance, people who suffer from quadriplegia, cervical spinal cord injury, or those who have suffered injuries in their arms and legs in an accident, may not be able to control the wheelchair via the joystick. Operating the existing manual or powered wheelchairs could be difficult or impossible for many of these individuals [46], since people with severe upper body motor impairment may not have sufficient hand functionality to use the joystick. To accommodate these severely disabled individuals and support their independent mobility, researchers developed a number of alternative wheelchair controls $[4,9,12$, $15,29,33,36,40,45,47]$.

In our previous work [27], we have presented a novel wheelchair control approach based on an egocentric camera. The key novelty in the design is that the wheelchair can be operated via the user's head motion, which is translated into the motion of a cursor on a tablet mounted in the user's field of view. In the prototype system, which is the one shown in this article, we have achieved satisfactory results using a web camera mounted on the user's hat, but even more discreet designs, such as a camera installed in eyeglasses, similar to the Google Glass, are also possible. To drive the wheelchair, users perform a slight head motion and the corresponding camera motion is tracked by observing a fiducial marker, also mounted on the wheelchair. This has the advantage that the environment does not need to be instrumented and that we do not need to compensate for the motion of the wheelchair. Tracked camera motion is mapped to cursor motion, which allows the user to control a virtual joystick and operate the wheelchair. Since the required range of motion is limited, the amount of required effort is small, and no external forces are applied to the neck of the user. The wheelchair is also equipped with other sensors, primarily a consumer depth camera (Microsoft Kinect), which support autonomous and semi-autonomous navigation [8, 28]. These functionalities, however, are not in scope here. The cost of all sensors and modifications to the commercially available powered wheelchair is much lower than the cost of the wheelchair itself.

In this article, we present three usability studies, which were conducted to evaluate the proposed system. In the first study, which was also presented as validation of the design of the system [27], 10 subjects performed a navigation task using two different modes of controlling the direction of the wheelchair: discrete and continuous. This comparison is of interest since many hands-free control alternatives, such as voice recognition, provide a set of discrete commands to the users. Our results show that continuous control of directions is preferable to discrete commands. 
In the second study, which has been published in a conference paper [25], 21 subjects were asked to compare the proposed vision-based control approach along with two baseline approaches: chin-based control, in which a modified mechanical joystick is placed below the user's chin so that the user can drive the wheelchair using head-motion, and manual control, in which the user operates a regular joystick. This study comprised two rounds, in which the subjects were required to navigate the wheelchair in an indoor test area using our vision-based approach and the two baseline approaches. We propose to establish joystick control as a reference that is available to all researchers in this area. By comparing their methods to manual control, the results of studies such as ours would be immediately comparable to each other. While we do not expect hands-free control methods to enable faster navigation times compared to joystick control, the loss of efficiency can be used to quantify the effectiveness of alternative methods.

The last part of the article, which is unpublished, presents a panel study to measure the improvement in subjects' performance when they are provided with carefully designed training sessions. Six subjects participated in four sessions each, following a training protocol while their navigation skills are assessed qualitatively and quantitatively. After completing initial easy tasks, we ask the subjects to navigate without the frontal display and in dynamic environments with other people. This third study consists of three phases. First, participants are placed in a training environment with a defined set of tasks to develop their wheelchair navigation skills. Second, the participants' performance and confidence in navigating the wheelchair using our hands-free approach is measured. Third, the participants' ability to navigate without the attached frontal display is assessed. Six subjects participated in this study in four one-hour weekly sessions.

Our quantitative and qualitative results show that the vision-based control approach is viable for hands-free indoor use. Moreover, the improvement in performance in round two of the second experiment using our method and the follow-up trials of the third experiment provide evidence that users can close the gap to joystick control with practice. The next steps are studies with individuals with disabilities and regular wheelchair users.

The main contributions of this work include:

(1) a natural and convenient human-robot interaction method using an egocentric camera;

(2) a qualitative and quantitative evaluation of the advantages a continuous over discrete control of direction;

(3) proposing the use of conventional joystick control as a reference for evaluating different approaches for wheelchair control leading to a well-understood, universal criterion, which will hopefully be adopted by the research community;

(4) a two-round user study indicating that the proposed vision-based method is viable, since it is only moderately slower than alternative approaches using mechanical devices, which may not be suitable for our intended users;

(5) an analysis of quantitative and qualitative results of the three studies that shows that user performance improves with time and that users gave more positive ratings to the visionbased control approach as they got more familiar with it.

\section{RELATED WORK}

Many hands-free wheelchair control methods have been developed as alternatives to manual control. Some of them have been commercially applied, while others are still confined in research laboratories. For an overview of these technologies we refer readers to surveys $[10,18,45,46]$. In this section, we focus on approaches in which the user controls the wheelchair at a low level by specifying the speed and direction of motion instead of giving high-level commands, such as "go 
to the bedroom." We also discuss prior work on usability studies on hands-free wheelchair control methods.

Sip-n-puff [10] is an early method for controlling a wheelchair by applying different pressure on a pneumatic tube via "sipping" and "puffing." Its advantage is that it can be used by severely disabled users with limited head-mobility, but it disrupts the user's breath and limits his or her communication with others. Methods based on the use of the chin or tongue were also among the first alternatives for controlling a wheelchair. In early work by Lipskin [29], a re-designed joystick is placed below the wheelchair user's chin, allowing the user to control the wheelchair. However, the customized joystick may still cause fatigue to the user's neck and facial muscles as it is a mechanical device on which the user must apply force. Tongue motion can be detected via contact with a small oral mechanical joystick, or more recently with electronic sensors, such as acoustic [33], inductive [32], and magnetic [19,23] sensors. The need, however, for the user to wear special mouthguards or externally visible magnets is a limitation of these methods.

Voice control is also among the hands-free solutions. Voice recognition technology [2, 14, 37], which requires only a microphone and a computer in terms of hardware, has recently demonstrated reliable performance in general. Users can utter short phrases, such as "move forward," to give commands. The challenges of this approach are, first, that the commands must be discrete leading to jagged behavior of the wheelchair, second, that the system may receive accidental commands during conversation, and, third, that there may be delays between speech and its interpretation.

Technologies that sense neuromuscular activation have also been investigated. For example, electromyography, which measures muscular activity [13, 17, 38, 47-49]; electrooculography, which measures eye movements [1,47]; and electroencephalography, which measures brain activity $[5,13,31,51]$ can all be used for detecting user's intention for wheelchair control. These methods have great potential to help severely disabled individuals with very limited mobility. However, only a very small number of discrete commands are available to the user and these interfaces require his or her full attention. Furthermore, mastering a brain-computer interface (BCI) requires extensive training over a period of weeks or months to generate stable volitional control [5].

Since our primary focus is to develop a hands-free solution for quadriplegic patients, research using head-motion as the input is of great interest to us. Head-motion is a natural way to control a wheelchair by mapping it to wheelchair motion. One technology for sensing head pose is via the use of tilt sensors, such as those found on most smartphones [9, 36].

Alternatively, head-motion can be measured by cameras. Vision-based approaches can be categorized as inward-facing, in which the camera is fixed on the wheelchair focusing the user's face $[20,21,41,43,50]$, and outward-facing or egocentric, in which the camera faces the environment $[16,22,27,52]$.

Jia et al. [20] map facial gestures to commands by tracking facial features of the user. Purwanto et al. [41] use the pan angle of the gaze and eye blinks to control the wheelchair. In a similar approach, Ju et al. [21] use the inclination of the user's face to determine the direction of the wheelchair and the shape of the user's mouth for moving forward and stopping. Rechy-Ramirez and $\mathrm{Hu}$ [43] detect four head motions and two facial gestures, which are converted to commands for the robotic wheelchair. Xu et al.'s wheelchair [50] receives input from the gaze of the user. Sensors mounted on the wheelchair, as well as markers and beacons placed in the scene, are combined for navigation and obstacle avoidance.

Research on outward-facing cameras is more recent due to the challenges associated with interpreting images of a dynamic environment under potentially unpredictable illumination. Halawani et al. [16] argue that an outward-facing camera is superior to an inward-facing one in terms of tracking resolution due to its wider field of view. They mount a web-camera on the user's hat and orient it downward to capture the user's clothes and the wheelchair so that the observed 
motion is due to head-motion rather than wheelchair-motion. Five discrete commands are activated based on the estimated motion of the web-camera. Kim et al. [22] present a robotic wheelchair equipped with only a pan-tilt-zoom (PTZ) camera. They utilize special visual markers, which have some black parts called black-peak that move according to the viewing angle. Hence, the robotic wheelchair can localize itself more accurately with the help of these special markers to complete challenging tasks such as passing through a door. Our method works well with a simple marker printed on paper.

Zolotas et al. [52] equip the wheelchair user with a Microsoft Hololens, which provides perception and visualization capabilities in one device. The see-through augmented reality display of the Hololens enables the system to attach virtual annotations to real objects in the field of view of the user to explain the behavior of the robot. The user controls the wheelchair manually via the joystick following a shared control paradigm. The sensors of the Hololens are used for localizing the user's head in space and for placing the virtual content appropriately, but not for controlling the wheelchair. The latter functionality was introduced by Chacón-Quesada and Demiris [7] who added an eye-gaze tracker that made several discrete, high-level commands available to the user.

The majority of previous hands-free mobility solutions such as the brain-computer interface, voice commands based control and face or head gesture based control provide discrete motion commands to drive the wheelchair. For example, a typical set of five motion commands can be LEFT, RIGHT, FORWARD, BACKWARD, STOP. In contrast, our method enables continuous control of direction and adjustment of speed. Computer vision based methods have advantages compared to other approaches [5, 14, 29, 30,37]. First, the low cost of web cameras makes these systems more affordable. Second, computer vision based methods do not require the users to physically operate any device and thus protect users from repetitive stress or repetitive motion injuries. Third, computer vision based methods enable users to hold conversations with others while operating the wheelchair.

In the second part of this section, we review usability studies of relevant hands-free technologies, especially focusing on methods that do not need users to physically contact a mechanical joystick. Many of the papers surveyed above include small usability studies with no more than 10 subjects. However, there are a few exceptions including the system of Ju et al. [21], which was tested on 34 subjects, half of whom were disabled, and the system of Ferreira et al. [13], which was tested on 25 subjects.

Parikh et al. [39] conducted a study on 50 subjects comparing three paradigms for navigating an intelligent wheelchair: a deliberate mode in which motion plans are made a priori based on maps and other information, a reactive mode in which obstacles are detected by the sensors and avoided using reactive controllers, and a manual mode in which the user drives using the joystick. Based on these modes three levels of operation were evaluated: autonomous control that combines the deliberate and reactive modes, manual control with the reactive controllers for collision avoidance, and semi-autonomous control that combines all three modes. The effort required by the user varies with the level of operation as expected; autonomous control leads to the fastest completion times, while semi-autonomous control is the slowest, but the differences are small.

Boucher et al. [2] conducted a usability study on a wheelchair design that enabled two autonomous control modes as well as a reactive control mode for users who could manipulate the input devices or give voice commands. The authors designed a robotic wheelchair that can be controlled in multiple ways, including via discrete commands given by voice or from the keyboard and via continuous commands given by a joystick. Seventeen individuals, including eight wheelchair users, participated in comprehensive experiments comparing the vocal interface to the joystick in various tasks. Subjects who were able to use the joystick were also able to achieve more precise control. 


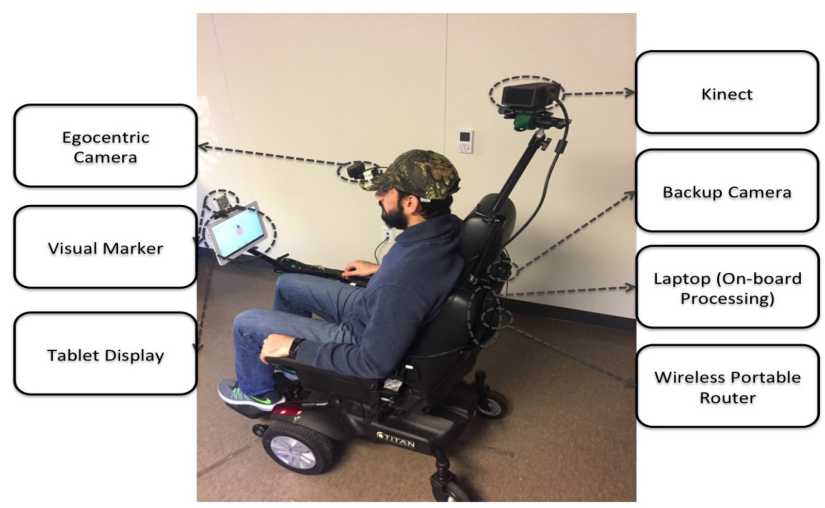

Fig. 1. The robotic wheelchair with the primary components labeled. The key sensor is the egocentric camera, which is used as a control device by the user whose head motion can be tracked as the egocentric camera tracks the visual marker, which is mounted on the robot. The Kinect is mainly used for localization, mapping, and obstacle avoidance in autonomous navigation mode that is out of the scope of this article. The tablet is a means of visualization, displaying navigation information to the user.

Wei et al. [49] published a usability study of a wheelchair control system relying on EMG signals and facial gesture recognition, to generate six discrete commands. Five users navigated a trajectory using this interface as well as using a joystick. They concluded that the proposed method is effective in controlling the wheelchair, but navigation times were at least three times longer compared to joystick-based control.

Candiotti et al. [3] compared conventional powered wheelchairs with their Mobility Enhancement roBotic (MEBot) wheelchair, which has six independently height-adjustable wheels for indoor and outdoor navigation. Twelve subjects with an average of 16.3 years of powered wheelchair driving experience participated in the study, which showed higher efficacy and safety of the MEBot, which come at the cost of higher mental demand on the users.

\section{ROBOTIC WHEELCHAIR DESIGN}

We briefly introduce our assistive robotic wheelchair. As shown in Figure 1, it is developed based on a powered wheelchair driven by a joystick (Drive Medical Titan Transportable Front Wheel Power Wheelchair). We emulate the electrical signals generated by the joystick with signals generated from an Arduino micro-controller. A Kinect sensor is mounted on the wheelchair looking forward over the user's head. The Kinect sensor is used for mapping and localization during autonomous navigation and for obstacle avoidance during autonomous and semi-autonomous navigation and is out of the scope of this article.

A tablet mount is set up to hold a tablet as a display device in front of the user. A visual marker is affixed on the tablet mount to facilitate camera pose estimation. A wearable camera is mounted on a baseball cap that is worn by the user. An additional backward-facing camera is attached to the back of the wheelchair, and its video stream is displayed on the tablet when moving backward. (The backward-facing camera was added before the third study.) The software system is built with the Robot Operating System (ROS) [42]. Figure 2 shows a diagram of our system.

\subsection{Head Motion Tracking}

As shown in Figure 3, head motion tracking uses a web camera mounted on the user's head and a visual marker mounted on the wheelchair. In the GUI, we make the motion of the cursor proportional to the camera motion, which is tracked as above. 


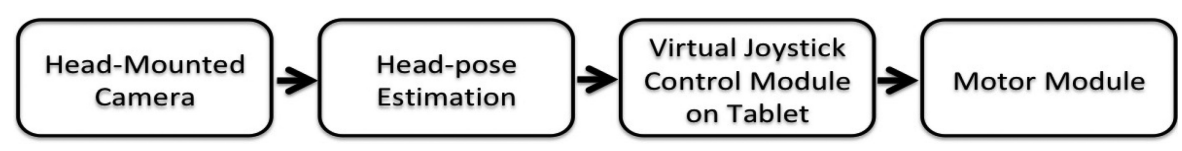

Fig. 2. Diagram of robotic wheelchair system. The arrows indicate data flow.

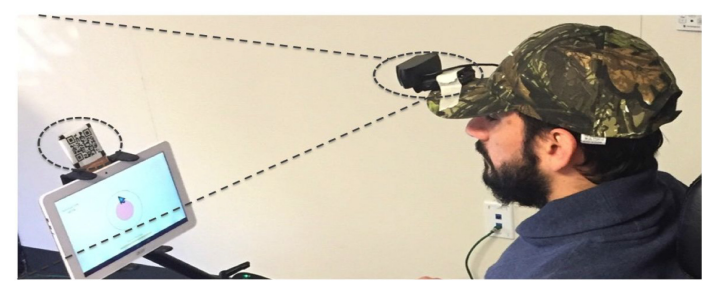

Fig. 3. The setup for head motion tracking. The camera and visual marker are highlighted.

To make the visual marker distinctive from common objects, we use a Quick Response (QR) code marker, the ViSP library [34] to detect the marker and the Consensus-based Matching and Tracking of Keypoints for Object Tracking (CMT) tracker [35] to track it. This approach has proven to be very robust in practice even in the presence of motion blur; there has been no complaint from the users that tracking was inconsistent or not responsive. By attempting to re-detect the marker in every frame, our system is able to recover from tracking errors quickly, before the corresponding commands to the cursor become noticeable. In Figure 5, we show four head poses that correspond to the maximum speed in each direction.

By tuning the ratio of camera motion to cursor motion on the screen, and due to the high precision in marker detection and tracking, small head motions are sufficient for controlling the wheelchair. As a result, operating the system requires little effort and does not cause strain to the neck.

\subsection{User Interface}

The proposed system provides a Graphical User Interface (GUI) on the tablet as shown in Figure 4. The head motion of the user moves a cursor on the GUI. Some pre-defined actions could invoke GUI events of the cursor. For example, a button click is invoked by hovering the cursor over a button for a pre-defined time length.

A typical work-flow with the proposed control includes the following steps:

(1) move the cursor to the "navigation mode" button, and keep the cursor on the button for 3 seconds to start manual control;

(2) move the cursor to the center, and keep it there until the center round button is "picked up" by the cursor;

(3) move the cursor to the target direction, and use the distance of the cursor to the center to control the speed;

(4) move the cursor back to the center to reduce the speed to zero, and keep it there until its color changes to "put back" the center round button;

(5) move the cursor to the "navigation mode" button, and keep the cursor on the button for 3 seconds to exit manual control.

As described above, this hands-free control design simulates the full functionality of a physical joystick so that it provides similar driving experience. Since the system accepts commands that are proportional to head motion, the user can easily give continuous commands to specify the 


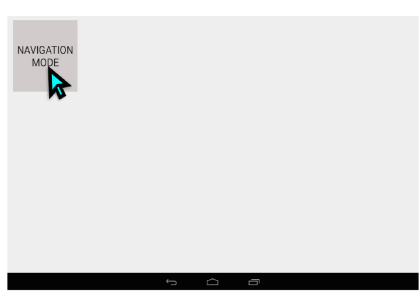

(a) Turn navigation on

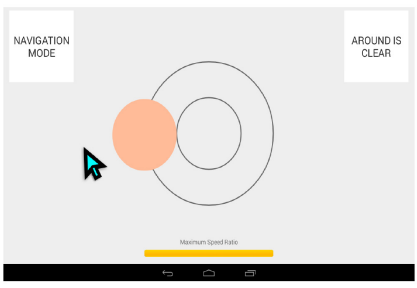

(d) Move left

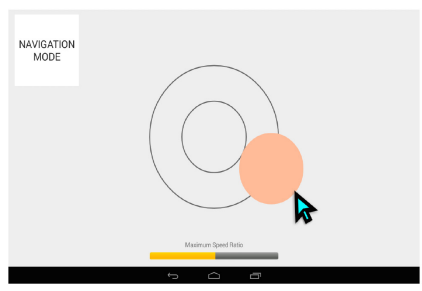

(g) Automatic brake

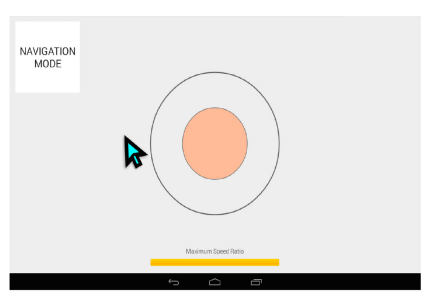

(b) Navigation GUI

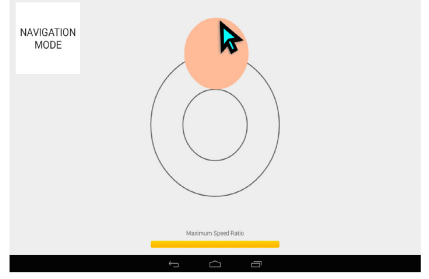

(e) Move forward

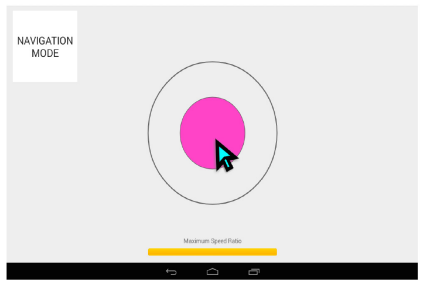

(h) Put back the virtual joystick

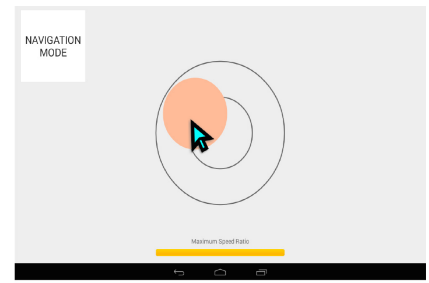

(c) Pick up the virtual joystick

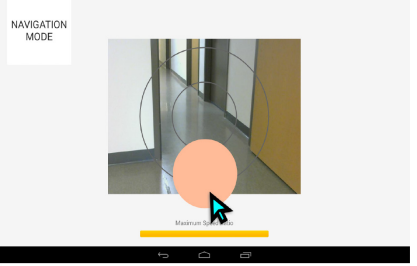

(f) Move backward - backup camera on

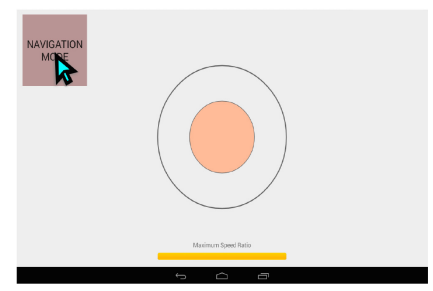

(i) Stop manual navigation

Fig. 4. The GUI of the robotic wheelchair.

direction and speed of the wheelchair. Moreover, the wheelchair can be used in any space since no external instrumentation or prior maps are required.

An example work-flow is shown in Figure 4. In our system, we include an automatic brake for safety in case the user is distracted during the manual navigation. As shown in Figure $4(\mathrm{~g})$, the bar below indicates the maximum speed of the wheelchair. When the visual marker is outside of the field of view of the wearable camera, the maximum speed decreases gradually to zero. For example, when the user looks away while driving the wheelchair, the wheelchair gradually applies the automatic brake to keep the user safe.

\section{USER STUDIES}

In this section, we present the three usability studies and the evaluation metrics used in these studies in accordance with the literature.

\subsection{Evaluation Metrics}

The metrics are selected according to the guidelines of Jia et al. [24]. Several papers on hands-free wheelchair control use similar metrics in their evaluations. Our selected metrics are the following:

(1) Navigation time for each section of the route. Navigation time reflects the user's skills and the ease of use of a particular control method. We record time per section since different sections of the route require different skills $[2,13,17,20,23,50]$. 


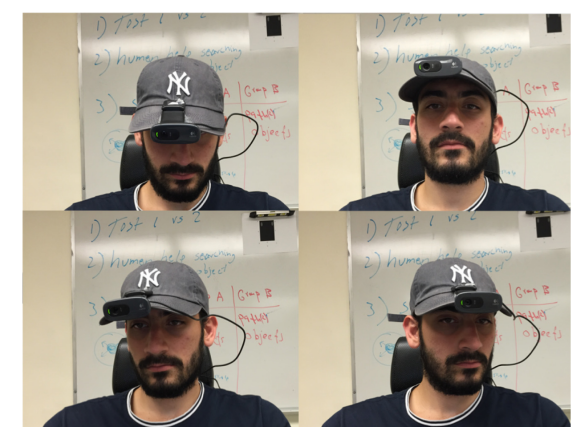

Fig. 5. The head poses giving maximum speed in different directions when continuous control is activated. The same poses correspond to the discrete commands when discrete control is evaluated in the first user study. Commands for forward, backward, right, and left are shown from top left to bottom right in clockwise order.

(2) Capability rating. This reflects the completeness of tasks preformed by subjects [24].

(3) Number of collisions. This reflects the safety of each mode [17, 20, 50].

(4) Surveys and comments. These complement the metrics with subjective evaluations [17].

(5) Practice time. Although not reported in previous publications, this metric reflects the subjects' own estimation of their skills in the respective control mode before the timed experiments.

\subsection{Continuous vs. Discrete Control of Direction}

A navigation task is designed to evaluate the usability of the proposed egocentric computer vision based control by comparing our system with its continuous control of direction to an alternative implementation of our system with four discrete direction commands. We assess both options quantitatively and qualitatively by recording navigation times and asking the subjects to complete questionnaires. We recruited 10 healthy participants, 9 male and 1 female, for this study among the student population at Stevens Institute of Technology.

4.2.1 Study Design. To enable the comparison of our proposed approach with previous solutions, we implemented a baseline control method, which provides five motion commands. One of the commands is to stop the wheelchair and it is activated by the neutral head pose. Two other commands drive the wheelchair forward and backward; these are activated by tilting the head up or down. The last two commands are to rotate the wheelchair left or right, and they are invoked by turning the head in the corresponding direction. These head motions are shown in Figure 5, and they are the same that trigger maximum-speed motion when the wheel chair is operated in continuous control mode.

We set up a navigation task in a corridor as shown in Figure 6. We place empty boxes in the scene as obstacles and ask participants to drive the wheelchair from the start-point to the end-point without running into an obstacle.

Before the experiments, we demonstrate the two control methods to all participants to help them learn to navigate the wheelchair. To reduce the influence of driving experience obtained during the experiments, we ask 5 out of the 10 participants to drive the wheelchair with continuous control of direction first and switch to discrete control after successfully accomplishing the first task. The other five participants preform the test with the discrete control first.

We use two metrics to evaluate the quality of the navigation: navigation time and number of attempts. We count how many times the subjects failed before they successfully accomplish the 


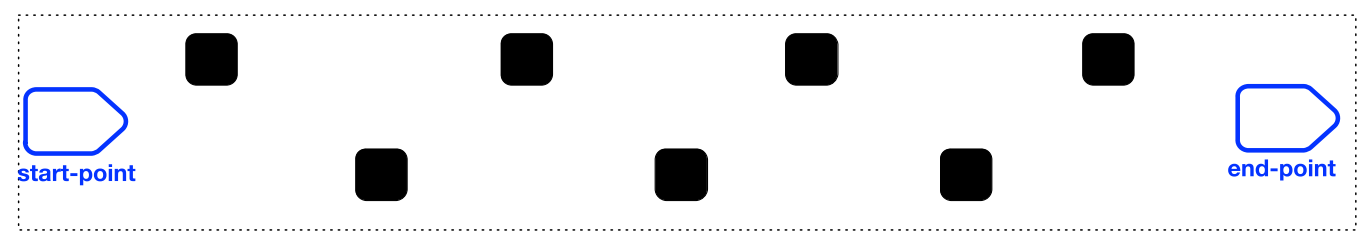

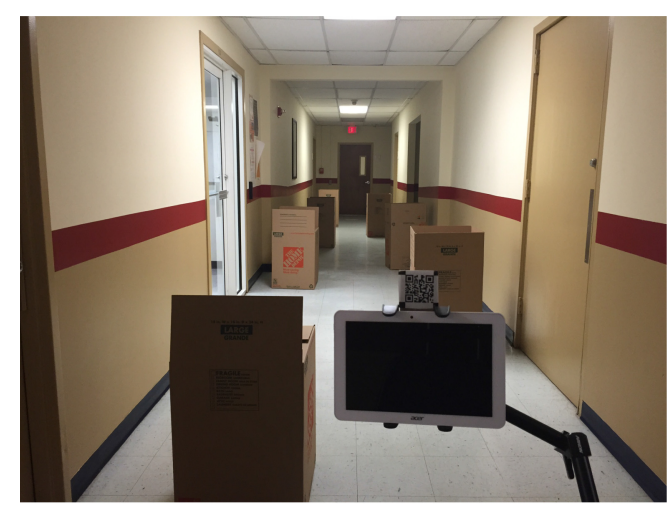

View from the start-point in the task

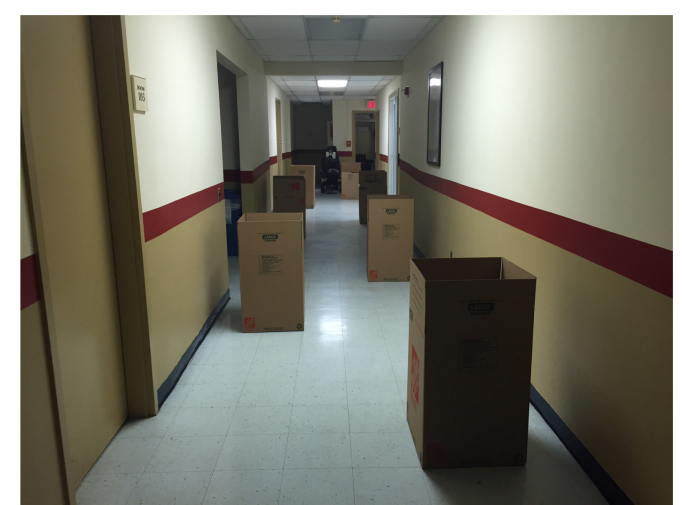

View from the end-point in the task

Fig. 6. The task: participants are asked to navigate from the start to the end point.

Table 1. The Questionnaire Given to the Participants Who Were Asked to Give a Score for How They Agree with Each Statement after Trying Each Control Method

\begin{tabular}{l}
\hline 1. It was easy to learn to use this control method. \\
2. I feel comfortable using this control method. \\
3. I like using the interface of this control method. \\
4. The control method is effective in helping me complete the tasks.
\end{tabular}

task. The wheelchair is autonomously stopped when it is about to hit an obstacle. When this happens during a navigation task, the navigation is regarded as a failure. The navigation time is recorded when the participant successfully accomplishes the task. In general, a control method is more difficult to learn when the number of attempts is large, but this does not indicate that the control method is hard to use. The user may take more than one attempt to learn a control method. However, once the users understand how to drive the wheelchair with a given control method, it can take them less time to accomplish the task. We observed this situation in our experiments with two participants.

The two metrics measure the usability of a control method objectively. In addition, we ask the participants to complete a questionnaire on their experience driving the wheelchair after they finish the task with each of the two control methods. We follow the Computer System Usability Questionnaire by Lewis et al. [26] to design the questionnaire. The participants are asked to report their agreement to the statements listed in Table 1 with a score between 1 and 7 in which 1 indicates strongly disagree while 7 indicates strongly agree. After they finish evaluating both control methods, we ask them to choose their preferred method.

4.2.2 Results. The experimental results are shown in Table 2. Most participants accomplish the task in the first attempt, which indicates that the head motion based control is intuitive. As we 
Table 2. Evaluation of the Control Methods in the Navigation Task

\begin{tabular}{c|c|c|c|c|c|c|c|c|c|c|c|c}
\hline & \multicolumn{10}{c}{ Metrics } \\
& \multicolumn{10}{|c}{ Questionnaire } & \multicolumn{1}{c}{ Questionnaire } \\
Participant & 1 & 2 & 3 & 4 & Nav. Time & Num. of Attempts & 1 & 2 & 3 & 4 & Nav. Time (s) & Num. of Attempts \\
\hline A & 7 & 6 & 7 & 7 & 65 & 1 & 6 & 5 & 7 & 3 & 175 & 3 \\
\hline B & 6 & 4 & 5 & 5 & 182 & 2 & 7 & 4 & 5 & 5 & 262 & 1 \\
\hline C & 6 & 6 & 5 & 7 & 63 & 1 & 4 & 4 & 5 & 4 & 190 & 1 \\
\hline D & 7 & 7 & 7 & 7 & 66 & 1 & 5 & 5 & 4 & 6 & 250 & 1 \\
\hline E & 7 & 7 & 7 & 6 & 68 & 1 & 5 & 4 & 7 & 4 & 245 & 1 \\
\hline F & 7 & 7 & 7 & 7 & 95 & 1 & 7 & 6 & 5 & 5 & 241 & 1 \\
\hline G & 7 & 7 & 7 & 7 & 71 & 1 & 7 & 7 & 4 & 5 & 180 & 1 \\
\hline H & 7 & 7 & 7 & 7 & 101 & 2 & 6 & 3 & 3 & 2 & 193 & 1 \\
\hline I & 7 & 6 & 5 & 6 & 80 & 1 & 7 & 5 & 6 & 5 & 209 & 1 \\
\hline J & 6 & 6 & 7 & 7 & 96 & 1 & 6 & 5 & 5 & 5 & 254 & 1 \\
\hline Average & 6.7 & 6.3 & 6.4 & 6.6 & 88.7 & 1.2 & 6 & 4.8 & 5.1 & 4.4 & 219.9 & 1.2 \\
\hline
\end{tabular}

observed, it takes the participants far less time to accomplish the tasks with the proposed continuous control of direction. Even for some participants, such as participant B, who requires two attempts to accomplish the task with continuous control, it takes him less time to finish the task with it in the second attempt compared to discrete control. On average, the participants agree that the proposed control method is easier, more comfortable to use, and more effective in completing the task than the baseline discrete control of direction.

\subsection{Chin-Based vs. Vision-Based Usability Study}

In this section, we present an evaluation for our vision-based control compared to joystick control as reference and also to chin-based control, which is an alternative hands-free control method that provides continuous control of direction and speed. We will refer to using the joystick as manual mode.

4.3.1 Study Design. Twenty-one healthy subjects ( 19 male and 2 female) were recruited for this study. Eleven of these subjects were between 20-29 years old; 10 were between $30-39$; and 1 was above 50. In each of the two rounds, the subjects were required to navigate through a test area using all three modes described in Section 4.3.2. Since previous studies have shown that familiarity plays an important role in such experiments and that subjects had little difficulty in manual mode, all subjects started the tests in manual mode using the physical joystick. After becoming familiar with the test area, the subjects were randomly split into two groups: the first group continued with chin-based and then with vision-based control, while the second group followed the opposite sequence.

The test area was set up in a hallway as shown in Figure 7. Obstacles, including chairs and boxes, were placed in the scene. Chairs are "see-through" obstacles (i.e., subjects were able to observe the scene behind them and be prepared in advance); boxes are opaque obstacles.

Before navigation in a given mode, each subject was given a brief introduction to it and was allowed to practice by navigating freely outside the test area. When subjects felt ready, they navigated the designated route. Practice time varies across subjects, but it serves as a proxy for familiarity with the user interfaces. 


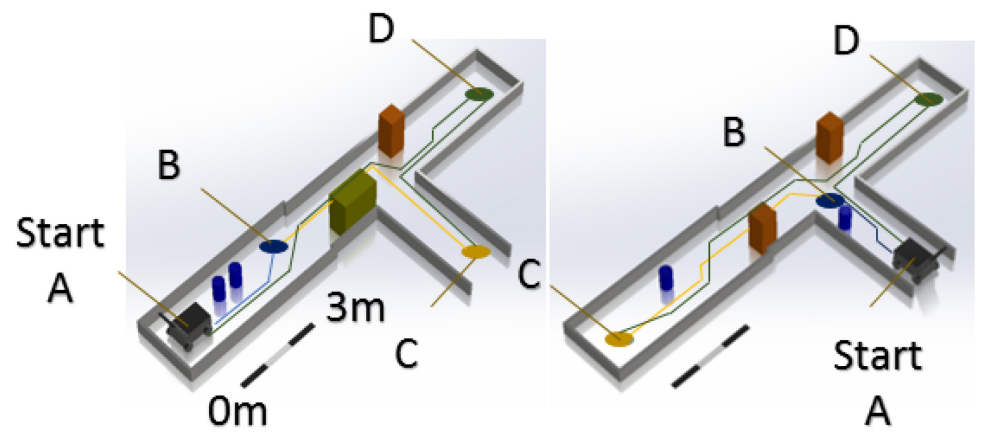

Fig. 7. Test area with approximate route. Left: first-round test; right: second-round test. Cylinders correspond to chairs (see-through obstacles) and boxes correspond to opaque obstacles.

Table 3. Sections of Designated Route

\begin{tabular}{lccl}
\hline Section & Round 1 distance & Round 2 distance & \multicolumn{1}{c}{ Note } \\
\hline $\mathrm{A} \rightarrow \mathrm{B}$ & $5.5 \mathrm{~m}$ & $4.5 \mathrm{~m}$ & Short distance navigation with forward-turn \\
$\mathrm{B} \rightarrow \mathrm{C}$ & $10 \mathrm{~m}$ & $11 \mathrm{~m}$ & Medium distance navigation and a $90^{\circ}$ turn-in-place \\
$180^{\circ}$ turn & $0 \mathrm{~m}$ & $0 \mathrm{~m}$ & $180^{\circ}$ turn-in-place in a narrow corridor \\
$\mathrm{C} \rightarrow \mathrm{D} \rightarrow \mathrm{A}$ & $25.3 \mathrm{~m}$ & $25.3 \mathrm{~m}$ & All of the above combined \\
\hline
\end{tabular}

The same set of subjects participated in the second round, which took place $2-5$ days later following the same procedure. The designated navigation route in the second round was different, but of comparable difficulty. Descriptions and dimensions of the test routes are listed in Table 3.

A questionnaire, shown in Table 8, was also given to the subjects after they completed the tests. Subjects were asked to answer each question in a 5-point Likert scale: strongly disagree, disagree, neither agree nor disagree, agree, and strongly agree. Results can also be seen in Table 8 .

4.3.2 Navigation Modes. In manual mode, the wheelchair is driven by a manufacturer-provided joystick. The joystick allows continuous control since the heading angle is read from the joystick and propagated to the control module as a continuous variable. This mode can only be used by users who do not suffer from upper-limb mobility impairments.

Chin-based control is among the oldest hands-free methods for driving a wheelchair [29]. In order to compare our vision-based method with other existing hands-free control methods, a customized chin-control device was made for the chin-based navigation mode. As shown in Figure 8, the chin-control mount has adjustable height to accommodate different users. To enable chinbased control, we customized the joystick provided by the manufacturer as shown in Figure 8 . The modified joystick still generates continuous linear and angular velocity commands. Similar to the vision-based mode, this mode can be used by users who suffer from upper-limb mobility impairments.

In vision-based mode, the user gives commands to the wheelchair by head-motion. The user only needs to wear a head-mounted camera to operate the wheelchair. This technique has been described in Section 3.

4.3.3 Analysis of Navigation Time. Table 4 shows the average time and standard deviation (SD) for each section in each navigation mode in the first round. Vision-based control has the longest 


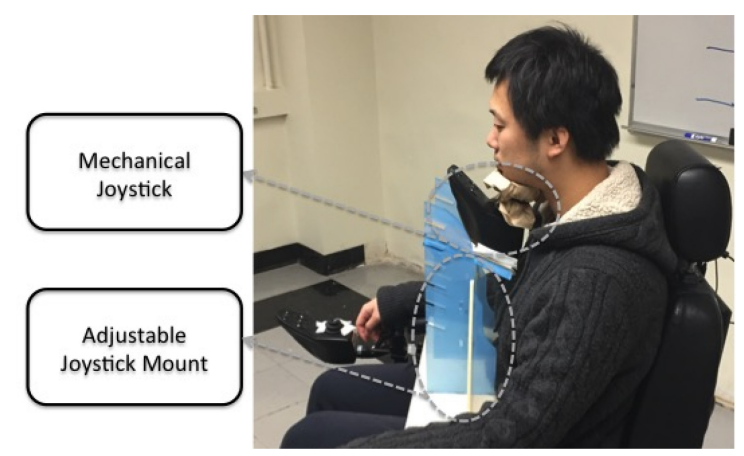

Fig. 8. The setup for chin-based mode.

Table 4. Practice and Navigation Time for the First Round

\begin{tabular}{lrrrrrr}
\hline \multirow{2}{*}{ Section } & \multicolumn{2}{c}{ Vision-based } & \multicolumn{2}{c}{ Chin-based } & \multicolumn{2}{c}{ Manual } \\
\cline { 2 - 7 } & Mean & SD & Mean & SD & Mean & SD \\
\hline Practice & 123.8 & 50.7 & 85.5 & 38.2 & 77.0 & 32.8 \\
$\mathrm{~A} \rightarrow \mathrm{B}$ & 14.7 & 4.3 & 11.8 & 2.5 & 11.2 & 3.1 \\
$\mathrm{~B} \rightarrow \mathrm{C}$ & 31.5 & 8.4 & 26.2 & 4.3 & 24.0 & 3.6 \\
$180^{\circ}$-turn & 11.7 & 4.9 & 6.1 & 1.5 & 6.2 & 1.3 \\
$\mathrm{C} \rightarrow \mathrm{D} \rightarrow \mathrm{A}$ & 86.3 & 16.2 & 71.9 & 9.2 & 64.7 & 5.3 \\
Total A $\rightarrow \mathrm{A}$ & 144.2 & 25.6 & 116.0 & 12.9 & 106.1 & 9.6 \\
\hline
\end{tabular}

Table 5. Practice and Navigation Time for the Second Round

\begin{tabular}{lrrrrrr}
\hline \multirow{2}{*}{ Section } & \multicolumn{2}{c}{ Vision-based } & \multicolumn{2}{c}{ Chin-based } & \multicolumn{2}{c}{ Manual } \\
\cline { 2 - 7 } & Mean & SD & Mean & SD & Mean & SD \\
\hline Practice & 49.3 & 46.8 & 40.4 & 32.9 & 35.1 & 29.9 \\
$\mathrm{~A} \rightarrow \mathrm{B}$ & 9.9 & 1.4 & 9.7 & 1.4 & 9.4 & 1.1 \\
$\mathrm{~B} \rightarrow \mathrm{C}$ & 28.1 & 7.6 & 23.2 & 2.5 & 22.3 & 1.7 \\
$180^{\circ}$-turn & 6.9 & 1.6 & 5.8 & 1.5 & 5.0 & 0.8 \\
$\mathrm{C} \rightarrow \mathrm{D} \rightarrow \mathrm{A}$ & 80.1 & 11.7 & 72.3 & 9.3 & 64.5 & 4.2 \\
Total A $\rightarrow$ A & 125.0 & 17.8 & 110.9 & 12.4 & 101.1 & 6.3 \\
\hline
\end{tabular}

practice time, the longest navigation time in most sections with few exceptions, and the longest overall time; not surprisingly, manual control has the shortest.

On average for the entire route navigated (excluding practice time), chin-based control is $19.6 \%$ faster than vision-based control, while manual control is $26.4 \%$ faster. From the results of the different sections, it can be seen that the biggest difference between the vision-based control and the other two baseline approaches is the $180^{\circ}$ turn-in-place: the time for turning using the vision-based control is almost twice as long as that for using chin-based or manual control.

Table 5 shows the average time and SD for the overall navigation time in the second round. Similarly to the first round, the mode with the slowest navigation is still the vision-based one. Manual navigation is still the fastest and chin-based control comes second. Manual navigation is $19.1 \%$ faster than the vision-based mode, while chin-based navigation is $11.3 \%$ faster. It appears that practice is more beneficial for the vision-based method since it is the least familiar form of 
Table 6. Change of Overall Navigation Time between Two Rounds

\begin{tabular}{lrrr}
\hline & Vision-based & Chin-based & Manual \\
\hline Absolute decrease in avg. time (s) & 19.2 & 5.1 & 5.0 \\
Relative decrease in avg. time (\%) & $13.3 \%$ & $4.4 \%$ & $4.8 \%$ \\
\hline Number of subjects whose navigation time... & & & \\
decreased & 18 & 14 & 12 \\
increased & 3 & 7 & 6 \\
stayed the same & 0 & 0 & 3 \\
\hline
\end{tabular}

Table 7. Number of Collisions in Both Rounds

\begin{tabular}{cccc}
\hline Mode & Vision-based & Chin-based & Manual \\
\hline Round 1 Major & 8 & 3 & 0 \\
Round 1 Minor & 6 & 5 & 2 \\
Round 2 Major & 2 & 0 & 0 \\
Round 2 Minor & 6 & 2 & 1 \\
\hline
\end{tabular}

control to the subjects. The difference in the $180^{\circ}$ turn-in-place is no longer as pronounced as in the first round, showing that subjects are able to learn how to execute specific maneuvers relatively fast. Four subjects chose to forgo the untimed practice in the second round for chin-based control, six for manual control, and seven for the vision-based control. These choices were not always linked with good performance in the test. It may be worth investigating whether they were due to over-confidence or discomfort with one or more navigation modes.

Table 6 shows a comparison of the two rounds in terms of overall navigation time, as well as in terms of the number of subjects whose navigation time increased, decreased, or remained constant. On average, for the vision-based control, the overall navigation time (excluding practice time) was reduced by 19.2 seconds (13.3\% relative to the first round).

4.3.4 Analysis of Safety. Collisions are categorized into "major" and "minor." We use the following specifications when recording collisions during the tests. We record a major collision when the subject needs to come to a complete stop and requires assistance. We record a minor collision when the wheelchair grazes an obstacle and the subject is able to correct the trajectory without external help. Table 7 lists the number of collisions. From the table, it can be seen that in the first round, subjects are able to avoid major collisions only using the manual control mode. There is a substantial improvement in all modes in the second round. The only major collisions occur in the vision-based mode, but for under $10 \%$ of the subjects.

4.3.5 Survey Results. The survey statements and results are listed in Table 8. A 5-point Likert scale is used, with 5 being strongly agree and 1 being strongly disagree. The results show that vision-based control receives lower ratings in all questions. The ratings, however, are positive for all methods. Considering that vision-based navigation requires the least physical effort, it can still be a realistic option for wheelchair control, especially for individuals with limited use of their hands.

The last question in the second round shows that the subjects have confidence in improving their vision-based navigation skill through practice. This matches what we have found from the analysis of navigation times.

Table 8 also shows how the subjects' opinion changed after testing all methods for the second time. Vision-based navigation has the lowest initial ratings, but it partially closes the gap with the 
Table 8. Survey Statements and Results

\begin{tabular}{lcrrrrr}
\hline \multirow{2}{*}{ Question/Round } & \multicolumn{2}{c}{ Vision-based } & \multicolumn{2}{c}{ Chin-based } & \multicolumn{2}{c}{ Manual } \\
\cline { 2 - 7 } & Mean & SD & Mean & SD & Mean & SD \\
\hline 1. My overall experience of this navigation method was satisfactory. \\
Round 1 & 3.7 & 0.8 & 3.6 & 1.0 & 4.9 & 0.3 \\
Round 2 & 3.9 & 0.6 & 3.9 & 1.0 & 4.7 & 0.9 \\
Increase \% & $5 \%$ & $-25 \%$ & $7 \%$ & 0 & $-3 \%$ & $200 \%$ \\
\hline
\end{tabular}

2. In this method it was easy to learn to operate the wheelchair.

\begin{tabular}{lrrrrrr} 
Round 1 & 3.9 & 0.9 & 4.1 & 1.0 & 5.0 & 0.0 \\
Round 2 & 4.1 & 0.7 & 4.1 & 0.9 & 5.0 & 0.0 \\
Increase \% & $4 \%$ & $-22 \%$ & 0 & $-10 \%$ & 0 & 0 \\
\hline
\end{tabular}

3. I felt safe when I navigated using this navigation method.

\begin{tabular}{lrrrrrr} 
Round 1 & 3.0 & 0.9 & 3.9 & 0.9 & 5.0 & 0.0 \\
Round 2 & 3.7 & 1.0 & 3.9 & 1.1 & 4.8 & 0.5 \\
Increase \% & $18 \%$ & $-11 \%$ & 0 & $22 \%$ & $-4 \%$ & $\mathrm{~N} / \mathrm{A}$ \\
\hline
\end{tabular}

4. I felt my performance improved, compared to the first time.

\begin{tabular}{lllllll} 
Round 2 & 4.3 & 1.1 & 3.9 & 1.0 & 4.3 & 1.1 \\
\hline
\end{tabular}

other modes in the second round, after the subjects have become somewhat more familiar with it. Manual navigation is still preferable to our healthy subjects, but it is not an option for the target population of our robot.

4.3.6 Discussion: Advantages of Vision-Based Method. Although the vision-based method showed higher collision rate and longer navigation time in the evaluation, it also showed several advantages:

(1) Minimal physical effort is needed in vision-based control. Chin-based control requires the users to continuously move their neck and head, while the mechanical joystick imposes forces back to the head and indirectly to the neck. In our experiments, we noticed that while using chin-based control, some subjects were fatigued around the end of the test navigation. A subject requested a break in the middle of the experiment while using the chin-based method.

(2) Performance can be greatly improved with practice in the vision-based control: as seen in the experimental results, the improvement in navigation time from the first to the second round is noteworthy in the vision-based method. Of course, it should be noted that user satisfaction was initially higher with the other methods and, as a result, improvement in their ratings is small. The survey results show that for the vision-based method, subjects' satisfaction improved noticeably in the second round compared to the first round. A subject commented: "I think I have already reached my best in round 1 for the chin-based and manual control; but using the vision-based control, my performance improved in round 2."

4.3.7 Discussion: Disadvantages of Vision-Based Method and Analysis. During the evaluation, we identified the following issues with the vision-based method:

(1) Some subjects showed less confidence in vision-based control than the other control methods: in round 2, some subjects referred to the vision-based as the "hard one."

(2) Subjects were less aware of the environment: subjects devoted most of their attention to the virtual joystick on the tablet, which prevented them from looking around. When 
Table 9. List of Questions Asked before the First Session

\begin{tabular}{l|l}
\hline Answer Type & \multicolumn{1}{c}{ Questions } \\
$\begin{array}{l}\text { Structured (yes/no) } \\
\text { Unstructured }\end{array}$ & $\begin{array}{l}\text { Have ever driven a wheelchair before? } \\
\text { If you have, what was the method of operation? Joystick, vision, tongue. } \\
\text { Unstructured } \\
\text { If you have, what was the purpose of driving? Test drive, driving for fun, } \\
\text { injury, or other reasons. } \\
\text { When and where did you see people use wheelchairs? On the Internet, social } \\
\text { media, hospital, or the street? }\end{array}$ \\
$\begin{array}{l}\text { Unstructured } \\
\text { Unstructured }\end{array}$ & $\begin{array}{l}\text { Wo you think a person with hand usage difficulties can navigate a wheelchair? } \\
\text { Please look at our room and the corridor. If you were asked to navigate inside } \\
\text { this room, what are the challenges you might encounter in the space? }\end{array}$ \\
\hline
\end{tabular}

the other methods are employed, users sense the position of the mechanical joystick by touching, leaving their eyes free to observe the surroundings. It should be noted, however, that no other alternatives may be available in some cases.

\subsection{Panel Study}

In this section, we presented a usability study with a small panel of six subjects who participate in multiple sessions of training and evaluation of their navigation skills. This study consists of three parts. First, participants are placed in a training environment with a defined set of training tasks to develop their wheelchair navigation skills. Second, the participants' performance and confidence to navigate using our hands-free wheelchair is measured. Third, the participants' abilities to navigate without the attached frontal display are assessed.

4.4.1 Study Design. Six healthy participants were recruited: three female and three male, between 25 and 35 years old. The study was conducted over 4 weeks, in 1-hour weekly sessions, labeled S1 through S4. It consists of a set of predefined navigation tasks that the subjects were asked to perform. Tasks were described to participants verbally during the experiment. In addition to the tasks, there was one pre-experiment and four post-sessions surveys. In the pre-experiment survey, all subjects answered "No" to the "Have you ever driven a wheelchair?" question, which indicates how limited their familiarity to wheelchairs was before the experiment. The first two sessions included navigation tasks to be performed with the frontal display enabled. The third session includes both tasks with and without the frontal display, while in the fourth session, the frontal display was removed from the wheelchair. All tasks were video-recorded for offline analysis. Some of the tasks were performed by subjects once and some were performed twice. We use the terms first and second trial to refer to the first and second session a given task was performed, regardless of the exact sessions the trials were performed in. In other words, a session for us refers to one of the four times a subject operated the wheelchair, while a trial is tied to a specific task and refers to an attempt by a subject to perform the task.

4.4.2 Surveys. Subjects were asked to complete a pre-experiment survey and four post-sessions surveys shown in Tables 9 and 10, respectively. The surveys include a mix of structured and unstructured questions. The pre-experiment survey aims to gauge the subjects' level of familiarity with wheelchairs and their opinions of hands-free solutions before the experiment. The postsession surveys focus on collecting data about the subjects' impression after every session as well as their suggestions for improving the system. 
Table 10. List of Post-Session Survey Questions

\begin{tabular}{l|l|l}
\hline ID & Answer Type & \multicolumn{1}{c}{ Questions } \\
\hline Q1 & Unstructured & What do you think the biggest challenges for people to use a wheelchair are? \\
Q2 & Structured & Do you think that a person without use of the hands can operate the wheelchair? \\
Q3 & Structured & How would you rate your overall satisfaction in the use of the wheelchair? \\
Q4 & Structured & How would you rate your overall satisfaction in the navigation of the wheelchair? \\
Q5 & Structured & Was adequate time given to practice how to use the hands-free wheelchair? \\
Q6 & Structured & Was it safe when you navigated the hands-free wheelchair? \\
Q7 & Structured & Did you feel that you have spent less time navigating the wheelchair after training? \\
Q8 & Unstructured & Please describe your experience in this week's training. \\
Q9 & Unstructured & Are there any additional comments? \\
\hline
\end{tabular}

Questions Q2 - Q7 are answered on a scale from 1 to 5.

Table 11. List of Tasks and Their Descriptions

\begin{tabular}{|c|c|}
\hline Tasks & Description \\
\hline Turn in place & Rotate the wheelchair 180 degrees \\
\hline Forward turn & Rotate the wheelchair 90 degrees while moving forward \\
\hline Maneuver & Move from a position on a line to the same position on a parallel line \\
\hline Door pass & Pass through a door \\
\hline 2m Backward & $\begin{array}{l}\text { Move backward in a straight line for } 2 \text { meters while remaining close to the } \\
\text { center of a lane or a corridor }\end{array}$ \\
\hline 10m Straight & $\begin{array}{l}\text { Move forward in a straight line for } 10 \text { meters while remaining close to the center } \\
\text { of a lane or a corridor }\end{array}$ \\
\hline $104 \mathrm{~m}$ navigation & $\begin{array}{l}\text { Navigate in a square, } 50 \text {-meter corridor, and then reverse direction and navigate } \\
\text { the same corridor; end the task with passing through a door. }\end{array}$ \\
\hline In-room navigation & Navigate inside a room: (a) door to desk, (b) desk to door, (c) door to refrigerator \\
\hline Dynamic environment & Navigate safely in an environment with people moving around \\
\hline
\end{tabular}

4.4.3 Navigation Tasks. In every session, subjects were instructed to perform a set of tasks listed in Table 11. Some of these tasks, which we refer to as basic navigation tasks, were taken from the Wheelchair Training Manual [24] while we have designed some longer navigation tasks, which we refer to as long navigation tasks. The long-navigation tasks are combinations of several basic-navigation tasks. For instance, the $104 \mathrm{~m}$ navigation task described in Table 11 consists of multiple navigation tasks such as Forward turn, 10m Straight, and Door pass. It should be noted that long-distance navigation, such as the $104 \mathrm{~m}$ task, is not particularly informative about the subjects' skill set [24]. Segments that require specific maneuvers are more valuable. As a result, we did not schedule the $104 \mathrm{~m}$ navigation task in the last session. We include it in Table 13 to show the gap in performance between the subjects and the expert.

4.4.4 Evaluation Criteria. To measure performance, we use qualitative criteria, namely the capability rating, which is based on the Wheelchair Training Manual and indicates the subject's skill level, and quantitative criteria, specifically the time required by a subject to finish a task. The capability ratings are assigned by the experimenters and range from zero to two, with zero indicating failure to complete the task, and two meaning that the subject was able to complete the task. The severity of collisions was also considered in the capability rating. For major collisions, a capability rating of zero would be recorded and, for minor collisions, a capability rating of one would be recorded. Major and minor collisions were defined in Section 4.3.4. Subjects were allowed to repeat 
the tasks until they achieve a capability rating of two. Capability ratings for all the attempts are considered in calculating a capability rating for the task.

We categorize subjects' sessions based on answers to unstructured questions of post-session surveys in four categories: Unsatisfactory, Basic, Good, and Excellent. Definitions of these categories and examples of subjects' answers are provided below.

Unsatisfactory means the subject gained no confidence after the training and rejected the use of the wheelchair without providing specific reasons. For instance, comments along the lines of "I did not feel any improvements in my training. Frankly, the wheelchair is very difficult to operate."

Basic means the subject gained little confidence after training and rejected the use of the wheelchair based on rational arguments. For instance, comments along the lines of "I feel more comfortable, but the only problem I faced today was when I lost control due to a software lag and hit the wall, while the wheelchair continued moving and hit the wall. It was scary; specifically, if I am close to a hill or stairs, I will be more afraid of losing control and hurting myself." Another subject commenting on the dynamic environment task stated that "Pedestrians do not bring trouble; however, it becomes much harder to navigate without the tablet." Yet another subject stated, "It is better than last time, but still kind of dangerous."

Good means the subject gained confidence after training and accepted the use of the wheelchair without providing specific reasons. For instance, a subject stated, "The training is more difficult which helps me navigate the wheelchair better," or "It is getting easier to control the wheelchair." Another example is a subject commenting on her performance saying "I think I did it better than last week, at least the training part." Moreover, a subject commented on the interaction with pedestrians that "I got a little nervous when I came across the pedestrian, but it is not too hard to deal with this problem."

Excellent means the subject felt confident after training, accepted the use of the wheelchair, and was able to critically review the training process, and show her motor skills development. For instance, a subject stated, "After practicing the first time, everything got much easier. It is very similar to driving a car, so I think a driver can learn to handle it much quicker." Moreover, a subject commented on the interaction with pedestrians that "It is not hard to pass by somebody; I just need to adjust the direction a little bit." Also, a comment on the need for tablet display such as, "Without the tablet, it was easier to focus on the surroundings and learn to use my eyes independently of my head motion. The backward motions were more difficult without the tablet because it provided backward camera view," would be classified as excellent.

The time required by subjects to finish a task is an indicator of how well the subject learns to perform the required tasks. Even though subjects were allowed multiple trials, only the last trial time is reported. To obtain an indication of the optimal navigation time, we ask an expert to drive the wheelchair using the regular joystick preforming all the navigation tasks, which subjects were asked to perform. The expert's navigation time serves as the optimal time.

4.4.5 Results. Tables 12 and 13 show the time and capability rating for the navigation tasks with and without the frontal display, respectively. Both tables have expert columns that show the performance of an expert using a physical joystick. Table 12 shows experimental results for tasks with the frontal display enabled. For five tasks, navigation times decreased in the second trial in comparison to the first trial, while only for two tasks, it did not. The total navigation time of all tasks slightly decreased in the second trial in comparison to the first trial. Comparing the subjects to the expert, the subjects in both the first and second session took only $28 \%$ longer to complete the same tasks, which shows that the system is intuitive. The capability ratings, for almost all tasks, increased in the second trial in comparison to the first trial, which indicates the subjects completed the task better while taking a similar amount of time. 
Table 12. Average Capability Ratings and Navigation Times for Tasks in which the Tablet was Enabled in Both First and Second Trials of the Same Task

\begin{tabular}{l|r|rr|rr}
\hline & Expert & \multicolumn{2}{|c|}{ First Trial (S1) } & \multicolumn{2}{|c}{ Second Trial (S2) } \\
\hline Tasks & Time & Time & Capability & Time & Capability \\
\hline Turn in place & 7.50 & 9.60 & 1.93 & 8.75 & 1.94 \\
Forward turn & 7.00 & 12.61 & 1.71 & 11.88 & 2.00 \\
Maneuver & 14.00 & 26.23 & 1.37 & 27.19 & 1.58 \\
Door pass & 4.50 & 9.03 & 1.71 & 7.66 & 2.00 \\
2m Backward & 8.00 & 12.16 & 1.44 & 11.00 & 1.75 \\
10m Straight & 25.00 & 31.25 & 1.87 & 33.50 & 2.00 \\
104m navigation & 260.00 & 309.56 & 1.95 & 298.79 & 2.00 \\
Total & 326.00 & 410.46 & 12.06 & 396.86 & 13.28 \\
Compared to expert & - & $25.90 \%$ & - & $21.73 \%$ & - \\
In-room navigation & 51.00 & 82.50 & 2.00 & - & - \\
Compared to expert & - & $61.76 \%$ & - & - & - \\
Dynamic environment & 17.00 & 26.50 & 1.83 & - & - \\
Compared to expert & - & $55.88 \%$ & - & - & - \\
\hline
\end{tabular}

Reference navigation times of an expert are also reported. These trials took place in sessions S1 and S2.

Table 13. Average Capability Ratings and Navigation Times for Tasks in which the Tablet was Removed in Both First and Second Trials of the Same Task

\begin{tabular}{l|r|rr|rr}
\hline & Expert & \multicolumn{2}{|c|}{ First Trial } & \multicolumn{2}{c}{ Second Trial } \\
\hline Tasks & Time & Time & Capability & Time & Capability \\
\hline Turn in place & 7.50 & 27.41 & 1.61 & 8.41 & 1.52 \\
Forward turn & 7.00 & 62.16 & 1.65 & 13.00 & 1.88 \\
Maneuver & 14.00 & 91.66 & 1.69 & 22.36 & 1.83 \\
Door pass & 4.50 & 27.50 & 1.75 & 9.69 & 1.69 \\
2m Backward & 8.00 & 28.00 & 1.73 & 11.75 & 1.75 \\
10m Straight & 25.00 & 35.33 & 1.66 & 36.50 & 1.91 \\
\hline Total & 66.00 & 272.06 & 10.66 & 101.71 & 10.58 \\
Compared to expert & - & $312.12 \%$ & - & $54.10 \%$ & - \\
104m navigation & 260.00 & 330.26 & 2.00 & - & - \\
Compared to expert & - & $27.02 \%$ & - & - & - \\
Dynamic environment & 17.00 & 27.20 & 1.96 & - & - \\
Compared to expert & - & $61.76 \%$ & - & - & - \\
\hline
\end{tabular}

Reference navigation times of an expert are also reported. These trials took place in sessions S3 and S4.

Table 13 shows experimental results for tasks in which the frontal display was removed. For all tasks, except for the $10 \mathrm{~m}$ straight navigation task, navigation time decreased significantly from the first to the second trial.

Looking at the progress subjects made from the first to the second trial, we observe different behavior with and without the frontal display. In the former case, navigation times in the first trial were $25.9 \%$ slower than those of the expert. There is a small decrease in the second trial that brings these times within $21.73 \%$ of the expert's times, while there is an improvement in capability ratings. We hypothesize that, as shown in the previous studies, there is a $20 \%$ gap in speed between 
Table 14. Average Responses to Structured, Post-Session Survey Questions Q2 to Q7, for All Sessions with and without the Frontal Display

\begin{tabular}{l|cc|cc}
\hline Availability of frontal display & \multicolumn{2}{|c|}{ Yes } & \multicolumn{2}{c}{ No } \\
Session & First (S1) & Second (S2) & First (S3) & Second (S4) \\
\hline Q2: reliability & 4.83 & 4.67 & 4.00 & 4.83 \\
Q3: usability & 3.83 & 3.83 & 4.00 & 3.83 \\
Q4: navigation & 3.83 & 4.00 & 3.83 & 4.17 \\
Q5: practice time & 5.00 & 4.67 & 4.67 & 4.83 \\
Q6: safety & 3.67 & 3.50 & 3.50 & 3.83 \\
Q7: training usefulness & 4.33 & 4.17 & 3.83 & 4.67 \\
\hline
\end{tabular}

See Table 10 for full questions.

Table 15. The Average

Classifications of Subjects' Answers

to Unstructured Questions of

Post-Session Surveys Based on the

Criteria Presented in Section 4.4.4

\begin{tabular}{cccc}
\hline Session & S2 & S3 & S4 \\
\hline Subject & & & \\
\hline 1 & Basic & Good & Good \\
2 & Basic & Good & Good \\
3 & Basic & Basic & Basic \\
4 & Basic & Basic & Good \\
5 & Good & Good & Good \\
6 & Good & Good & Good \\
\hline
\end{tabular}

Only Sessions 2, 3, and 4 are shown because a prior session is required for classification.

vision-based and manual navigation, and that our subjects have reached their final speed quickly and improve in precision in the second trial. In experiments without the display, subjects start very far from the expert's speed and improve dramatically from the first to the second trial-from $312 \%$ to $54.1 \%$ slower than the expert. Their capability ratings remain constant since, apparently, they do not start mastering the skills required to navigate without the display in only two trials.

Subjects' navigation time for the dynamic environment tasks in the presence of people in the scene was about $55 \%$ slower than the expert's with the tablet and $61 \%$ without the tablet.

Table 14 shows the answers for structured questions in each post-session survey. It shows that subjects expressed more positive attitudes toward the system in the fourth session compared to the third one. On the other hand, in the experiments with the frontal display enabled, subjects expressed a slightly less positive attitude toward the system after the first trial than after the second trial. In general, the overall attitude of the subjects was positive toward the system and training tasks.

Table 15 shows our classifications for the subjects' answers to unstructured questions in postsession surveys, based on the criteria presented in Section 4.4.4. Based on the classifications, all subjects indicated that their skills were improving with the designed training. The table also shows that the amount of learning from each session is increased with practice, except for Subject 3, whose responses were classified as Basic in every session. 


\section{CONCLUSIONS}

We have presented and evaluated a novel approach for wheelchair control using a wearable egocentric camera that allows hands-free operation of the wheelchair. Three user studies were conducted to test the usability and safety of the system. Thirty-seven subjects participated in those experiments and several evaluation metrics were considered. The results of the first study show the effectiveness of having continuous over discrete control of direction. The results of the second study show that our proposed system is viable and safe for indoor navigation. We argue that the loss of speed due to camera-based control compared to operating the wheelchair using the joystick is acceptable considering that our objective is to make wheelchair operation possible for subjects with limited or no hand mobility. In the third study, we observed that the subjects' performance improved after training. This was reflected in their navigation times, capability ratings, survey answers, as well as in that they were quickly able to learn to navigate the wheelchair without the display. The limitation of our current work is that all subjects were able-bodied. These studies have established the viability of our approach. Our future efforts will focus on studies with subjects who rely on wheelchairs for their daily lives.

\section{ACKNOWLEDGMENTS}

All authors, except for Xiaxue Du, were at Stevens Institute of Technology when they contributed to this effort. The authors are grateful to Biruk Gabre, who is with Department of Mechanical Engineering at Stevens Institute of Technology, for manufacturing the chin-based control device.

\section{REFERENCES}

[1] Rafael Barea, Luciano Boquete, Manuel Mazo, and Elena López. 2002. System for assisted mobility using eye movements based on electrooculography. IEEE Transactions on Neural Systems and Rehabilitation Engineering 10, 4 (2002), 209-218.

[2] Patrice Boucher, Amin Atrash, Sousso Kelouwani, Wormser Honoré, Hai Nguyen, Julien Villemure, François Routhier, Paul Cohen, Louise Demers, Robert Forget, and Joelle Pineau. 2013. Design and validation of an intelligent wheelchair towards a clinically-functional outcome. Journal of Neuroengineering and Rehabilitation 10, 1 (2013), 1-16.

[3] Jorge L. Candiotti, Deepan C. Kamaraj, Brandon Daveler, Cheng-Shiu Chung, Garrett G. Grindle, Rosemarie Cooper, and Rory A. Cooper. 2019. Usability evaluation of a novel robotic power wheelchair for indoor and outdoor navigation. Archives of Physical Medicine and Rehabilitation 100, 4 (2019), 627-637.

[4] Tom Carlson, Robert Leeb, Ricardo Chavarriaga, and José del R. Millán. 2012. The birth of the brain-controlled wheelchair. In Proceedings of the IEEE/RSf International Conference on Intelligent Robots and Systems. 5444-5445.

[5] Tom Carlson and Jose del R. Millan. 2013. Brain-controlled wheelchairs: A robotic architecture. IEEE Robotics and Automation Magazine 20, 1 (2013), 65-73.

[6] Ramon Ceres, Jose L. Pons, Leopoldo Calderon, Antonio R. Jimenez, and Luis Azevedo. 2005. A robotic vehicle for disabled children. IEEE Engineering in Medicine and Biology Magazine 24, 6 (2005), 55-63.

[7] Rodrigo Chacón-Quesada and Yiannis Demiris. 2018. Augmented reality control of smart wheelchair using eye-gazeenabled selection of affordances. In Robots for Assisted Living workshop.

[8] Yizhe Chang, Mohammed Kutbi, Niklaos Agadakos, Bo Sun, and Philippos Mordohai. 2017. A shared autonomy approach for wheelchair navigation based on learned user preferences. In Proceedings of the 5th International Workshop on Assistive Computer Vision and Robotics. 1490-1499.

[9] Show-Hong Chen, Yu-Luen Chen, Ying-Han Chiou, Jen-Cheng Tsai, and Te-Son Kuo. 2003. Head-controlled device with M3S-based for people with disabilities. In International Conference of the IEEE Engineering in Medicine and Biology Society, Vol. 2. 1587-1589.

[10] Albert M. Cook and Janice Miller Polgar. 2014. Assistive Technologies: Principles and Practice. Elsevier Health Sciences.

[11] Linda Fehr, W. Edwin Langbein, and Steven B. Skaar. 2000. Adequacy of power wheelchair control interfaces for persons with severe disabilities: A clinical survey. Journal of Rehabilitation Research and Development 37, 3 (2000), 353-360.

[12] Torsten Felzer and Bernd Freisleben. 2002. HaWCoS: The hands-free wheelchair control system. In Proceedings of the 5th International ACM Conference on Assistive Technologies. 127-134. 
[13] Andre Ferreira, Wanderley C. Celeste, Fernando A. Cheein, Teodiano F. Bastos-Filho, Mario Sarcinelli-Filho, and Ricardo Carelli. 2008. Human-machine interfaces based on EMG and EEG applied to robotic systems. Fournal of Neuroengineering and Rehabilitation 5, 1 (2008), 1-15.

[14] Mohamed Fezari and Mounir Bousbia-Salah. 2007. Speech and sensor in guiding an electric wheelchair. Automatic Control and Computer Sciences 41, 1 (2007), 39-43.

[15] J. O. Gray, Pei Jia, Huosheng H. Hu, Tao Lu, and Kui Yuan. 2007. Head gesture recognition for hands-free control of an intelligent wheelchair. Industrial Robot: An International fournal 34, 1 (2007), 60-68.

[16] Alaa Halawani, Shafiq Ur Réhman, Haibo Li, and Adi Anani. 2012. Active vision for controlling an electric wheelchair. Intelligent Service Robotics 5, 2 (2012), 89-98.

[17] Jeong-Su Han, Z. Zenn Bien, Dae-Jin Kim, Hyong-Euk Lee, and Jong-Sung Kim. 2003. Human-machine interface for wheelchair control with EMG and its evaluation. In International Conference of the IEEE Engineering in Medicine and Biology Society, Vol. 2. 1602-1605.

[18] Tuck-Voon How, Rosalie H. Wang, and Alex Mihailidis. 2013. Evaluation of an intelligent wheelchair system for older adults with cognitive impairments. fournal of Neuroengineering and Rehabilitation 10, 1 (2013), 90-105.

[19] Xueliang Huo, Jia Wang, and Maysam Ghovanloo. 2008. A magneto-inductive sensor based wireless tongue-computer interface. IEEE Transactions on Neural Systems and Rehabilitation Engineering 16, 5 (2008), 497-504.

[20] Pei Jia, Huosheng H. Hu, Tao Lu, and Kui Yuan. 2007. Head gesture recognition for hands-free control of an intelligent wheelchair. Industrial Robot: An International fournal 34, 1 (2007), 60-68.

[21] Jin Sun Ju, Yunhee Shin, and Eun Yi Kim. 2009. Intelligent wheelchair (IW) interface using face and mouth recognition. In Proceedings of the 14th International Conference on Intelligent User Interfaces. ACM, 307-314.

[22] Bong Keun Kim, Hideyuki Tanaka, and Yasushi Sumi. 2015. Robotic wheelchair using a high accuracy visual marker LentiBar and its application to door crossing navigation. In Proceedings of the IEEE International Conference on Robotics and Automation. 4478-4483.

[23] Jeonghee Kim, Hangue Park, Joy Bruce, Erica Sutton, Diane Rowles, Deborah Pucci, Jaimee Holbrook, Julia Minocha, Beatrice Nardone, Dennis West, Anne Laumann, Eliot Roth, Mike Jones, Emir Veledar, and Maysam Ghovanloo. 2013. The tongue enables computer and wheelchair control for people with spinal cord injury. Science Translational Medicine 5, 213 (2013), 166-187.

[24] R. L. Kirby, C. Smith, K. Parker, M. McAllister, J. Boyce, P. W. Rushton, F. Routhier, K. L. Best, B. Mortenson, and A. Brandt. 2015. Wheelchair Skills Training Program (WSTP) Manual, version 4.3. Retrieved from http://www. wheelchairskillsprogram.ca/eng/documents/WSTP_Manual_4.1.50.pdf.

[25] Mohammed Kutbi, Yizhe Chang, and Philippos Mordohai. 2017. Hands-free wheelchair navigation based on egocentric computer vision: A usability study. In IROS Workshop on Assistance and Service Robotics in a Human Environment. IEEE.

[26] James R. Lewis. 1995. IBM computer usability satisfaction questionnaires: Psychometric evaluation and instructions for use. International fournal of Human-Computer Interaction 7, 1 (1995), 57-78.

[27] Haoxiang Li, Mohammed Kutbi, Xin Li, Changjiang Cai, Philippos Mordohai, and Gang Hua. 2016. An egocentric computer vision based co-robot wheelchair. In Proceedings of the IEEE/RSF International Conference on Intelligent Robots and Systems. IEEE, 1829-1836.

[28] Haoxiang Li, Philippos Mordohai, and Gang Hua. 2016. Attention-driven egocentric computer vision for robotic wheelchair navigation. In Proceedings of the 4th Workshop on Egocentric (First-Person) Vision. IEEE.

[29] Ronald Lipskin. 1970. An evaluation program for powered wheelchair control systems. Bulletin of Prosthetics Research 6 (1970), 121-219.

[30] Ana C. Lopes, Gabriel Pires, and Urbano Nunes. 2012. RobChair: Experiments evaluating brain-computer interface to steer a semi-autonomous wheelchair. In Proceedings of the IEEE/RSF International Conference on Intelligent Robots and Systems. IEEE, 5135-5136.

[31] Ana C. Lopes, Gabriel Pires, and Urbano Nunes. 2013. Assisted navigation for a brain-actuated intelligent wheelchair. Robotics and Autonomous Systems 61, 3 (2013), 245-258.

[32] Morten Enemark Lund, Henrik Vie Christiensen, Héctor Caltenco, Eugen Romulus Lontis, Bo Bentsen, and Lotte N.S. Andreasen Struijk. 2010. Inductive tongue control of powered wheelchairs. In Proceedings of the International Conference of the IEEE Engineering in Medicine and Biology Society. 3361-3364.

[33] Michael Mace, Khondaker Abdullah-Al-Mamun, Ravi Vaidyanathan, Shouyan Wang, and Lalit Gupta. 2010. Real-time implementation of a non-invasive tongue-based human-robot interface. In Proceedings of the IEEE/RSf International Conference on Intelligent Robots and Systems. IEEE, 5486-5491.

[34] Eric Marchand, Fabien Spindler, and François Chaumette. 2005. ViSP for visual servoing: A generic software platform with a wide class of robot control skills. IEEE Robotics \& Automation Magazine 12, 4 (2005), 40-52.

[35] Georg Nebehay and Roman Pflugfelder. 2014. Consensus-based matching and tracking of keypoints for object tracking. In Proceedings of theIEEE Winter Conference on Applications of Computer Vision (WACV). IEEE, 862-869. 
[36] H. T. Nguyen, L. M. King, and Glenn Knight. 2004. Real-time head movement system and embedded linux implementation for the control of power wheelchairs. In Proceedings of the International Conference of the IEEE Engineering in Medicine and Biology Society. 4892-4895.

[37] Masato Nishimori, Takeshi Saitoh, and Ryosuke Konishi. 2007. Voice controlled intelligent wheelchair. In Proceedings of the Society of Instrument and Control Engineers (SICE) Annual Conference. 336-340.

[38] Yuusuke Oonishi, Sehoon Oh, and Yoichi Hori. 2010. A new control method for power-assisted wheelchair based on the surface myoelectric signal. IEEE Transactions on Industrial Electronics 57, 9 (2010), 3191-3196.

[39] Sarangi P. Parikh, Valdir Grassi, Vijay Kumar, and Jr. Jun Okamoto. 2005. Usability study of a control framework for an intelligent wheelchair. In Proceedings of the IEEE International Conference on Robotics and Automation. 4745-4750.

[40] Elisa Perez, Carlos Soria, Natalia M. López, Oscar Nasisi, and Vicente Mut. 2013. Vision based interface applied to assistive robots. International fournal of Advanced Robotic Systems 10 (2013), 116-125.

[41] Djoko Purwanto, Ronny Mardiyanto, and Kohei Arai. 2009. Electric wheelchair control with gaze direction and eye blinking. Artificial Life and Robotics 14, 3 (2009), 397-400.

[42] Morgan Quigley, Ken Conley, Brian Gerkey, Josh Faust, Tully Foote, Jeremy Leibs, Rob Wheeler, and Andrew Y. Ng. 2009. ROS: An open-source robot operating system. In ICRA Workshop on Open Source Software. IEEE, 1-6.

[43] Ericka Janet Rechy-Ramirez and Huosheng Hu. 2014. Head movement and facial expression-based human-machine interface for controlling an intelligent wheelchair. International fournal of Biomechatronics and Biomedical Robotics 3, 2 (2014), 80-91.

[44] Margot Shields. 2004. Use of wheelchairs and other mobility support devices. Health Reports 15, 3 (May 2004), 37-41.

[45] Richard C. Simpson. 2005. Smart wheelchairs: A literature review. fournal of Rehabilitation Research and Development 42, 4 (2005), 423-436.

[46] Richard C. Simpson, Edmund F. LoPresti, and Rory A. Cooper. 2008. How many people would benefit from a smart wheelchair? fournal of Rehabilitation Research and Development 45, 1 (2008), 53-72.

[47] Chun Sing Louis Tsui, Pei Jia, John Q. Gan, Huosheng Hu, and Kui Yuan. 2007. EMG-based hands-free wheelchair control with EOG attention shift detection. In Proceedings of the IEEE International Conference on Robotics and Biomimetics (ROBIO). IEEE, 1266-1271.

[48] Lai Wei, Huosheng Hu, and Kui Yuan. 2008. Use of forehead bio-signals for controlling an intelligent wheelchair. In Proceedings of the IEEE International Conference on Robotics and Biomimetics (ROBIO). IEEE, 108-113.

[49] Lai Wei, Huosheng Hu, and Yi Zhang. 2011. Fusing EMG and visual data for hands-free control of an intelligent wheelchair. International Journal of Humanoid Robotics 8, 04 (2011), 707-724.

[50] Ruofei Xu, Robin Hartshorn, Ryan Huard, James Irwin, Kaitlyn Johnson, Gregory Nelson, Jon Campbell, Sakire Arslan Ay, and Matthew E. Taylor. 2016. Towards a semi-autonomous wheelchair for users with ALS. In IfCAI Workshop on Autonomous Mobile Service Robots.

[51] Rui Zhang, Yuanqing Li, Yongyong Yan, Hao Zhang, Shaoyu Wu, Tianyou Yu, and Zhenghui Gu. 2016. Control of a wheelchair in an indoor environment based on a brain-computer interface and automated navigation. IEEE Transactions on Neural Systems and Rehabilitation Engineering 24, 1 (2016), 128-139.

[52] Mark Zolotas, Joshua Elsdon, and Yiannis Demiris. 2018. Head-mounted augmented reality for explainable robotic wheelchair assistance. In Proceedings of the IEEE/RSF International Conference on Intelligent Robots and Systems. 18231829.

Received January 2019; revised March 2020; accepted May 2020 